\title{
Distinguishing Models for ACIS Data of Diffuse Emission
}

\author{
D.A. Leahy \\ University of Calgary, Alberta T2N 1N4, Canada
}

\begin{abstract}
Discussed here is the issue of discriminating different spectral models for diffuse emission observations with the Chandra ACIS. First background models are considered. Then standard source models such as nonionization equilibrium and ionization equilibrium models and models with different metal abundances are considered. The datasets used are from Chandra ACIS observations of diffuse emission, in particular of the Cygnus Loop supernova remnant.
\end{abstract}

\section{Introduction}

Here the issue of modeling of $\mathrm{x}$-ray spectra of diffuse $\mathrm{x}$-ray emission is studied. With the availability of data from new instruments like the Chandra ACIS, moderately good spectral resolution studies of diffuse emission can be carried out. Previous to Chandra and XMM, only low spectral resolution data of diffuse emission was available. Dispersed spectra of diffuse emission are not yet very useful, since the spatial extent of the diffuse emission severely compromises the spectral resolution.

In this study, diffuse emission from the Cygnus Loop taken with the Chandra ACIS is studied to determine what are the limitations of current data in determining the physical properties of the diffuse x-ray emitting gas. A bright region on the south- western limb is seen in ROSAT PSPC observations of the Cygnus Loop with a high hardness ratio filament directly adjacent to a low hardness ratio filament (Aschenbach \& Leahy 1999). This region was observed with the Chandra ACIS, with a field that was able to cover both filaments using the ACIS CCDs I0, I1, I2, I3 as well as cover a background region outside the Cygnus Loop with CCDs S2 and S3. The background was analyzed, then the source region was divided into several sub-regions for modeling of the diffuse emission spectrum. The use of data from a relatively old supernova remnant limits the discussion to models of thermal emission from low temperature gas $(k T<\sim 1 \mathrm{keV})$.

\section{Observations and data analysis}

A $10 \mathrm{ks}$ exposure of the southwest V region was obtained on 2000 May 21 with the Chandra ACIS. The field of view is roughly $17 \mathrm{arcmin}$ by $25 \mathrm{arcmin}$, and included the data from the six ACIS CCDs I0, I1, I2, I3, S2 and S3. All data processing was carried out using the CIAO2.3 package. Fig. 1 (left) shows a 

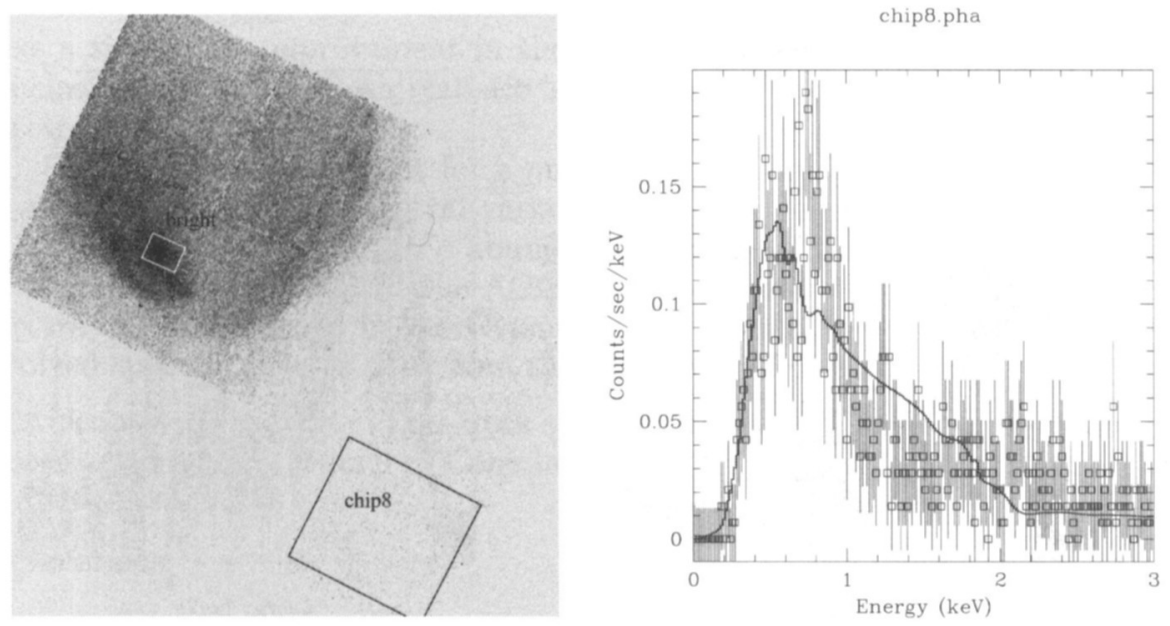

Figure 1. The Chandra ACIS image of the SW Cygnus Loop (left). The ACIS spectrum of the background region fit with the Gendreau et al. (1995) model (right).

processed image with north up. The image covers the energy band 0.3 to 1.4 $\mathrm{keV}$ and includes destreaking and exposure map corrections.

\subsection{Background data analysis}

A large region on ACIS S3 chip (as shown in Fig.1, left, labelled "chip8") was selected to yield the background spectrum. The background fitting was first carried out using pha channels 1-600 $(\sim 0-8.7 \mathrm{keV})$. Since the data for regions including Cygnus Loop emission did not show any excess above background above $\sim 2 \mathrm{keV}$, the background fitting was redone to better match this energy range, i.e. over pha channels $1-210(\sim 0-3 \mathrm{keV})$. The results for the background modeling were not significantly different. The fitting was done by minimizing the Cash statistic, $C=-2 \times \ln ($ likelihood $))$.

The initial model for the background was based on the results of Gendreau et al. (1995). They modeled the x-ray background for ASCA data as a sum of two power laws, with spectral indices of 1.37 and 3.90 and a thermal plasma emission with temperature $0.16 \mathrm{keV}$. This best fit model (normalizations free) is shown in Fig. 1 (right) compared to the data: it is clearly not adequate, being systematically too low from 0.6 to $1 \mathrm{keV}$ and systematically to high from 1 to $1.6 \mathrm{keV}$.

Next the mekal model (Kaastra 1992), was used to replace the xsraymond model for thermal plasma emission. To improve the systematics in the residuals it was necessary to free the power law index of the flat power law and the temperature. The result is shown in Fig. 2 (left). To improve the fit in the $0.5-0.8 \mathrm{keV}$ range, the oxygen abundance was made a free parameter. This resulted in a significant improvement in the $0.5-0.8 \mathrm{keV}$ residuals and gave zero amplitude to the steep power law with $\gamma=3.9$. Thus the steep power law was removed from the model. The resulting parameters were $\gamma=0.051, \mathrm{kT}=0.19$ $\mathrm{keV}$ and $\mathrm{O}$ abundance $=0.175 \times$ solar. This fit is shown in Fig. 2 (right). Freeing 

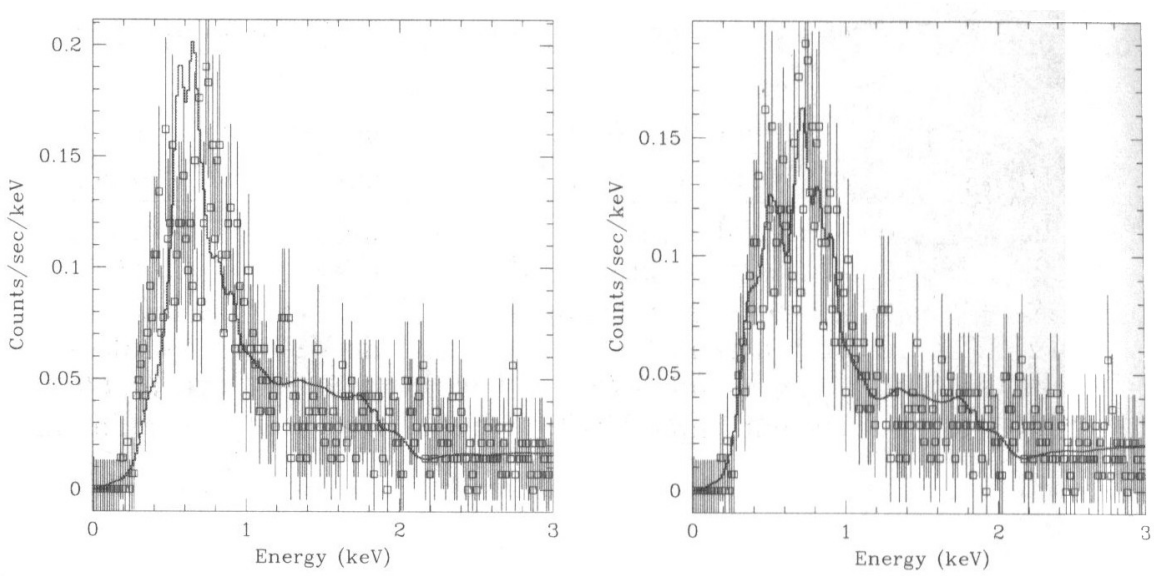

Figure 2. The ACIS spectrum of the background region fit with modified model (see text) (left). The ACIS spectrum of the background region fit with final background model (right).

abundances of other elements, including iron and neon, were tested but did not result in any significant improvement to the fit. Thus the final background model for the ACIS was determined.

\subsection{Comparison of source models using ACIS spectra}

The Cygnus Loop emission was spatially divided into a number of regions for spectral analysis. The brightest region (shown in Fig. 1 left) of those selected for spectral analysis was chosen as a test case for comparing source models. The best fit mekal model had $\mathrm{kT}=0.23 \mathrm{keV}$ and normalization of $0.0038 \times 10^{-14} /\left(4 \pi D^{2}\right) \int n_{e} n_{H} d V$. The fit is systematically bad $0.6-0.8 \mathrm{keV}$ and around $0.9 \mathrm{keV}$. Freeing the oxygen abundance greatly improves the fit (by $\simeq 15 \sigma$ ). Freeing the neon abundance further improves the fit (by $\simeq 7 \sigma$ ). Freeing the iron abundance also improves the fit (by $\simeq 6 \sigma$ ). However, freeing other element abundances individually does not further significantly improve the fit $(<3 \sigma)$. Freeing the hydrogen column density gave an improvement of $3.6 \sigma$. Allowing non-equilibrium ionization did not further improve the fit.

\section{Discussion}

The availability of data from the different ACIS CCDs has allowed a diffuse background and a diffuse source data analysis to be carried out on the same dataset. An improved background model was derived, which has allowed a better modeling of the source data.

During the fitting process no binning of spectral channels was carried out. The fitting was done by minimizing the Cash statistic. However the $\chi^{2}$ statistic was also computed for every fit. In several cases the improvement in the Cash statistic was significant but the improvement in $\chi^{2}$ statistic was not. At the same 
time a significant improvement in the fit residuals could be seen by eye. The conclusion is that the Cash statistic is more powerful than $\chi^{2}$ in distinguishing spectral fits.

The fits were carried out for a number of separate spatial regions. For the Cygnus Loop all of the spectral variation could be accounted for by variations in $k T, N_{H}$, and $\mathrm{O}, \mathrm{Ne}$ and $\mathrm{Fe}$ abundances. None of the spectra required any non-equilibrium ionization. The ACIS data has proven sufficient to distinguish spectra of small regions in the Cygnus Loop, whereas earlier data of spatially resolved spectra, such as that from the ROSAT PSPC, could not.

Acknowledgments. This work supported by the Natural Sciences and Engineering Research Council of Canada.

\section{References}

Aschenbach, B., Leahy, D., 1999, A\&A, 341, 602

Gendreau, K., Mushotsky, R., Fabian, A. et al. 1995, PASJ, 47, L5

Kaastra, J. S. 1992, An X-Ray Spectral Code for Optically Thin Plasmas, (Internal SRON-Leiden Report, updated version 2.0) 